\title{
Scribes, EHRs, and Workflow Efficiencies in Allergy Practices
}

\author{
Annette F. Carlisle ${ }^{1} \cdot$ Saul M. Greenbaum ${ }^{1} \cdot$ Mike S. Tankersley $^{1,2,3}$ (D) \\ Published online: 9 July 2020 \\ (C) Springer Science+Business Media, LLC, part of Springer Nature 2020
}

\begin{abstract}
Purpose of Review In the ever-changing healthcare system, along with new advancements in the field of allergy, the workflow for the allergist continues to evolve requiring more time spent doing non-clinical duties such as documentation and reviewing reimbursement challenges in the midst of busy clinics. The use of electronic medical records and medical scribes has emerged as tactics to aid the clinic's workflow and efficiency in the modern allergy and immunology clinic.

Recent Findings The practicing allergist can implement various additional strategies in their office workflow to maximize and synthesize good medicine and good business. Optimal use of office staff, electronic health records, and various workflow efficiencies has been shown to improve job satisfaction and reduce physician burnout.

Summary By utilizing these methods and integrating them into their practices, allergists will be able to meet the demands of the healthcare system and still provide patients with evidence based, compassionate, and cost-effective care.
\end{abstract}

Keywords Scribes · Electronic health records · Workplace efficiencies · Workflow

\section{Introduction}

As healthcare in this country continuously evolves and as insurance requirements take their toll on physicians' time and resources, allergy practices find themselves grasping for new and innovative ideas to keep ahead. Stress in the clinical setting has multiple sources, and perhaps chief among them is the clerical burden. Allergists need to find ways to lower this burden in order to continue to provide exceptional evidencebased medical care while minimizing physician burnout. This paper delineates several strategies for mitigating the clerical workload allergists' face. Electronic health records (EHRs) have existed in some form for over 50 years and present an opportunity to aid the physician's search for efficiency by

This article is part of the Topical Collection on Telemedicine and Technology

Mike S. Tankersley

mtanker6@uthsc.edu

1 Department of Pediatrics, Division of Pulmonary, Sleep, Allergy and Immunology, University of Tennessee Health Science Center, Memphis, TN, USA

2 Departments of Medicine and Otolaryngology, University of Tennessee Health Science Center, Memphis, TN, USA

3 The Tankersley Clinic, Memphis, TN, USA providing a standardized, multi-faceted, legible way to store all components of the patient record in one easily accessible, central location. Other recent developments in clinical productivity include the use of scribes as well as telemedicine that can exist in various shades of engagement within the office setting. By implementing these changes in allergy clinic routines and practices, along with other workplace efficiency improvements, the physician can carry the load of a modern practice and still deliver quality care and service to patients.

\section{Electronic Health Records}

The AAAAI's practice management resource guide defines electronic health records (EHRs) as an electronic record containing data about a patient that is integrated across multiple systems [1]. The existence of EHR dates back to the 1960s and 1970s when academic institutions developed their own EHR system. The development of EHR originally centered on the idea of creating a standardized method to compile patient information [2]. Over the last several years, the USA has put policies into place such as the Health Information Technology for Economic and Clinical Health (HITECH) Act of 2009 to incentivize providers to implement and utilize the EHR in a manner to promote meaningful use [3]. Subsequently, since 2015, Medicare-eligible professionals 
who do not participate in EHR meaningful use risk payment adjustment in their Medicare reimbursement [1]. Consequently, utilization of EHR is becoming a necessity for those practicing in the USA.

The EHR system exhibits multiple advantages including standardization of complete patient records in a legible format that may also be accessible to providers utilizing the same EHR system. Goals of the EHR system include (1) increased patient safety, decreased medication errors; (2) enhanced, rapid, effective communication; (3) reduced costs; and (4) improved quality of patient care through the organization of longitudinally obtained patient data [4]. Most research on EHR systems focuses on clinical, organizational, and societal benefits [5].

The clinical advantages of using EHR are vast including improving patient safety through mechanisms such as clinical decision support (CDS) systems and computerized physician order entry system (CPOE) [5]. Systems such as these can decrease medication errors as well as improve compliance rates in areas such as immunizations. A study by Bates et al. noted that serious medication errors reduced by $55 \%$ with use of CPOE alone [6]. A follow-up study by the same group demonstrated that with $\mathrm{CPOE}$ and CDS, serious medication errors decreased by $83 \%$ [7]. With e-prescribing, medication allergy alert notifications, drug interaction cross-check which combines both CDS and CPOE systems, a 2011 physician workflow study, which includes representatives of US office-based physicians, noted that $65 \%$ of responders felt that they were made aware of potential medication error through EHR [8•].

Other advantages include improvement in rates of adherence to clinical guidelines as demonstrated through a variety of clinical scenarios. One study in an outpatient rheumatology setting found that influenza and pneumococcal vaccine rates in immunocompromised patients improved from 47 to $65 \%$ and 19 to $41 \%$ respectively with the use of CDS systems integrated into the EHR [9]. Similar improvement in adherence to clinical guidelines regarding inpatient DVT prophylaxis and vaccination rates has been demonstrated $[10,11]$.

EHR systems can also lead to improved patient/provider communication by using secured messaging features (patient portal) to convey results, discuss treatment therapies, or answer questions. Makam et al. surveyed Texas PCPs on perception of EHR and found that $72 \%$ of 146 respondents felt that EHR helped foster electronic communication with patients [12]. Naturally, some providers might show hesitancy in using EHR due to the perception that it will negatively affect patient/provider communication. A systematic review by Alkureishi and colleagues reviewed 22 studies to explore the impact of EHR on the patient-provider relationship and communication. Results showed that 16 studies noted no change with EMR use, 5 demonstrated EMR use had positive impact and 1 study showed mixed results [13].
Furthermore, there has been published data specifically in the allergy/immunology literature that evaluates how EHR can further improve clinical practice. A study by Ziegler et al. found that by using EHR, real-time outreach to patients with excessive short acting beta agonist (SABA) refills led to a decrease number of SABA refills in the following year as well as sooner referral to allergy specialist [14]. Blumenthal and colleagues published a perspective article on how redesigning the allergy module in the EHR could lead to clarifying recorded drug allergies to better identify patients with true drug allergy and to help with management recommendations [15].

Organization benefits of utilizing EHR include more accurate and complete records leading to improved patient charge capture and reduced billing errors [5]. Additionally, EHR can help with patient notifications such as appointment, bill, or vaccine/immunotherapy reminders, decreasing the need for staff to perform these tasks. The paperless EHR model decreases the need for staff to file or physically pull paper charts [5]. By utilizing EHR, there is reduced paper use that not only decreases cost but also is more beneficial for the environment.

Societal benefits of EHR include creating a robust cohort for both clinical research and quality improvement measures. Use of EHR for clinical research has been demonstrated in observational studies to randomized control trials, and further research is ongoing to evaluate methods to expand this field in a safe way in order to adhere to patient privacy $[16,17]$. Use of EHR has also been evaluated in medical student education. In one study, researchers quantitatively characterized medical students' expectations of an EHR system in a hospital setting and impact on learning. Results showed that medical students recognized clinical advantages, did not consider their ability to interact with patients impaired, and thought EHR marginally improved feedback from clinicians [18].

Overall implementation of EHR has shown widespread advantages which has been shown in meta-analysis reviews in both the USA and Europe. The 2011 physician workflow study, compromised of outpatient physicians, noted that $78 \%$ of physician responders felt EHR enhanced patient care overall and $81 \%$ felt it helped access a patient's chart remotely. Thirty to $50 \%$ of respondents felt that EHR use was associated with clinical benefits related to recommended care, order of appropriate tests, and improved patient communication. This survey also found that physicians with longer EHR experience were most likely to report benefits [8•].

Some providers have historically shown hesitation towards implementation of EHR due to cost concerns. Historically costs associated with EHR have included not only implementation costs such as purchasing and installing hardware/software, converting paper charts to electronic and training users, but also ongoing maintenance costs and decreased revenue due to temporary loss of productivity while implementing the system. One survey in 2010 indicated that $25 \%$ of allergists reported concern over the cost of EHR as the main reason 
for not using an EMR system [1] and initial research estimated an average initial cost of \$50-70,000 per physician for a three person practice [5]. However, one study evaluating the EHR impact on workflow and financial measures found that staffing and practice expenses increased initially after EHR implementation but recovered to/close to pre-implementation levels after 12 months [19]. However, as the EHR has evolved with advancing technology and easier accessibility through Web-based programs, costs associated have decreased substantially. Cost-effective EHRs exist that can be purchased for less than $\$ 1200$ per year per provider. For example, one web based EHR has no start up fee with a $\$ 99$ per month maintenance fee [20].

Attributes of a good EHR encompass a wide variety of features including basic functions such as the ability to document all aspects of the patient encounter from demographic information, medication allergies, current medications, vital signs, list of past and current diagnosis, as well as imaging and lab results [4]. Other attributes of an optimal EHR based on physician perceptions would include ways to incorporate a consistent template for office visits, e-prescribing capabilities, patient portal secure messaging, e-fax, and a method for easy communication with referring providers to update on patient clinical status. Allergy/Immunology specific attributes include recording allergy skin testing documentation, immunotherapy dose customization, administration, and documentation, incorporation of extract ordering, interface with pulmonary function testing as well as integrating questionnaires such as asthma control test (ACT) [1].

\section{Scribes}

\section{Advantages and Cost Savings}

The use of medical scribes has emerged as a tactic to aid the clinic's workflow and efficiency. Multiple specialties have studied and analyzed the use of scribes from a qualitative and quantitative standpoint. In one oncology study in 2018, 33 physicians were paired with scribes in the clinical setting. The patient visit durations were reviewed compared with durations prior to the use of scribes in that clinic. There was a $12.1 \%$ decrease in the overall patient visit duration compared to previous clinic visits without scribes [21].

In addition to increased efficiency in the office, other specialties have measured increased productivity stemming from the use of scribes in their office. A 2017 study in a large outpatient urology office suggests that productivity as measured by both office E\&M (evaluation and management) visits as well as total RVUs (relative value unit) is significantly improved by the addition of scribes [22]. In that study, billable encounters were retrospectively reviewed. Number of billable encounters, scribe expense, revenue, and net revenue were compared between the first quarter of 2015 and the first quarter of 2016. The average encounters per physician increased by 152 , and average revenue increased by about $\$ 16,000$. The average scribe expense per physician per quarter was roughly $\$ 6000$ in that specialty setting. Thus, visit duration, office revenue, and billable encounters all improved with the addition of a cost effective use of scribes. Notable in this study, other staff already being utilized for other tasks in the office was not used as scribes. Optimal use of office staff and proper delegation, as in this case, of tasks and administrative work has been shown as well to improve job satisfaction and reduce physician burnout [23].

In one of the largest and most comprehensive studies on the impact of scribes in the medical workplace, Walker et al. [24•] prospectively studied the productivity of emergency room physicians over a 3-year period between November 2015 and January 2018 in five large medical centers in Australia. Physicians were randomized to shifts with a scribe versus shifts without a scribe. The five emergency rooms all differed in their characteristics, such as regional versus urban, to better extrapolate the results to a wider swath of physicians. The primary outcome of the study was the difference in physicians' productivity between scribed and non-scribed shifts. Other secondary outcomes were door-to-doctor times and length of stay times in the ER, among others. The presence of scribes was associated with productivity gains, as measured by mean number of patients per hour per physician. Across all the centers studied, physicians making use of a scribe saw roughly 0.21 patients more per hour than physicians not making use of a scribe. The secondary outcome results were more impressive, as overall, the patients overseen by physicians with a scribe averaged 19 min less length of stay compared to non-scribed physicians. As well, the study's cost-benefit analysis showed a cost saving to the hospital per scribed hour of US\$26.15. It is reasonable to deduce that similar improvements in patient length of stay, reduced cost per patient, and other productivity gains, can be achieved in the outpatient medical setting of an allergy/immunology clinic.

In the above-quoted study, Walker et al. had scribes accompany their allocated physician for the whole shift. The scribe used either an in-room computer or a computer-on-wheels to document consultations while the physician assessed the patient. Scribes also participated in other clerical duties. Physicians edited and verified scribes' documentation as well. In the upcoming section, we will discuss this and other variants of scribes day to day and minute to minute task in the office.

\section{Costs, Requirements, and Variants of Scribes}

Little data exists as to the average cost of scribes that are utilized in the outpatient setting across all medical specialties. There are cost figures scattered across the spectrum of 
outpatient specialties, however. In an outpatient academic urology setting, scribes were obtained at a cost of $\$ 77$ per session [25]. However, the cost was offset by the increased in physician charges per clinic day of over $\$ 500$, so the net financial impact was approximately $\$ 429$ per clinic session, resulting in more than a sixfold return on investment for that clinic. Other specialties noted similar costs of a scribe for a single clinical session, including an outpatient cardiology clinic [26] that studied increased academic physician satisfaction rates when using scribes. The above costs from those studies did not include the increased expenditures of those respective clinics for new equipment or electronic devices required when hiring a new scribe.

In the above-cited study by Pozdnyakova et al., scribes were required to undergo 40 hours of training on medical terminology and compliance, as well as 4 hours of EHR ambulatory training. Scribes shadowed each physician for one clinic session as well. Outpatient practices not involved in rigorous academic or research studies would doubtless require far fewer hours of training before the scribe could be transitioned into the practice.

Multiple scribe arrangements and task delegations exist or could potentially be implemented. Many medical personnel types can work as a scribe, including nurses, medical students, college students, or medical assistants. Scribe services may also be contractually arranged with a scribe company [27]. Ostensibly, the tasks performed can vary according to the individual clinical setting. There are four scribe settings, two involving participation in the clinical session and two not participating in the clinical session. Specifically, the scribe might participate in the clinical encounter by (1) going in to take the history before the provider or (2) going in with the scribe annotating while the provider takes the history. A scribe might also not participate in the clinical encounter but work in the clinic or remotely by (1) taking the provider's handwritten template notes and entering them into the medical note or (2) via a dictated note entered by a transcriptionist. Our clinic has successfully and effectively used scribes in all four settings as in Table 1. Shultz et al. reviewed five studies and found examples of scribes entering diagnoses, revising problem lists, completing follow-up request forms, typing patient instructions, documenting level of services and completing after-visit summaries. In other studies, scribes even were utilized to track laboratory and imaging tests, cross-check consultations, and follow admission requests [28]. Having staff work at the top of their training level helps to reduce clinic costs and increase efficiency. To our knowledge, no studies at this point have compared or contrasted the different variations of scribes or which variation leads to the greatest cost savings or efficiency improvements.

Another commonly utilized scribe method is the use of voice recognition software in the office setting. Benefits of medical transcription via voice recognition include long-
Table 1 Methods to integrate scribes into practice

\begin{tabular}{lcc}
\hline Type of scribe & Possible advantages & $\begin{array}{l}\text { Possible } \\
\text { disadvantages }\end{array}$ \\
\hline $\begin{array}{l}\text { 1) Additional history } \\
\text { collection before } \\
\text { provider sees the } \\
\text { patient. }\end{array}$ & $\begin{array}{c}\text { Provider can focus } \\
\text { on other tasks. }\end{array}$ & $\begin{array}{c}\text { Scribe is not trained } \\
\text { in a proper medical } \\
\text { history taking. }\end{array}$ \\
$\begin{array}{l}\text { 2) } \\
\begin{array}{l}\text { Annotation/Transcript- } \\
\text { ion of medical notes in } \\
\text { the room with provider }\end{array}\end{array}$ & $\begin{array}{c}\text { Real time } \\
\text { transcription of the }\end{array}$ & $\begin{array}{c}\text { Narrative form of } \\
\text { transcription may } \\
\text { be taken rather } \\
\text { than a proper } \\
\text { medical history }\end{array}$ \\
$\begin{array}{l}\text { 3) Insertion of provider's } \\
\text { template note into the } \\
\text { medical note. Scribe } \\
\text { does not enter patient } \\
\text { room. }\end{array}$ & $\begin{array}{c}\text { Provider can focus } \\
\text { on seeing the next } \\
\text { patient while the } \\
\text { scribe works on } \\
\text { the documentation }\end{array}$ & $\begin{array}{c}\text { Scribe is not present } \\
\text { to document while } \\
\text { in the exam room. }\end{array}$ \\
$\begin{array}{l}\text { 4) Insertion of provider's } \\
\text { dictated note into the } \\
\text { medical note }\end{array}$ & $\begin{array}{c}\text { As above. } \\
\text { Time consuming for } \\
\text { the provider. }\end{array}$ \\
\hline
\end{tabular}

term cost savings and nearly instant training of the software to the provider's needs. Additionally, as this method functions completely independent of office personnel, staff lunch breaks or vacation time will not affect workflow. On the other hand, medical transcription software cannot offer the same in person services as provided by a full-time scribe namely, the ability to take a preliminary history prior to the entry of the provider or to transcribe the provider's encounter with the patient in real time.

The decision as to which method or variation of scribe integration to implement is left up to the individual provider's style of practice. Some providers may feel relieved of the clerical burden if their scribe takes on as much responsibility as possible. Others may feel they want to continue to participate in the documentation process as in the past.

\section{Telemedicine}

The COVID-19 pandemic has accelerated the implementation of telemedicine into US medicine at an unprecedented rate. Even prior to the COVID-19 pandemic, telemedicine afforded allergists a way to improve workflow efficiency and extend the geographical reach of the practice without increasing overhead. Telemedicine can be a convenient setup for both provider and patient as it allows the patient encounter to occur virtually anywhere and can occur outside of normal business hours if needed [29]. While there are many telemedicine platforms available, free services are available that are functional and user-friendly for both the patient and provider [30]. Additionally, all documentation from the telemedicine visit can be entered into the existing EHR just as a face-to-face encounter would be documented. Most EHRs will even allow 
the provider to design a template specifically for a telehealth visit if needed. Furthermore, scribes can be utilized in this setting by inputting demographic information, medications, or allergies prior to visit and can continue to document the encounter in the EHR while the allergist is conducting the visit.

\section{Workflow Efficiencies}

Alongside utilization of medical scribes and the efficient use of EHRs, our outpatient allergy clinic has applied multiple principles and efficiencies to streamline patient throughput and to reduce overall waste of resources. We have always maintained that good medical practices and good business practices can and should coexist, without allowing the latter to dictate the terms of the former. The practicing allergist can and should have various strategies in their office workflow to maximize and synthesize good medicine and good business.

First, the allergist/immunology (A/I) specialist needs to be more involved with basic immunization practices by screening and providing recommended vaccinations to adolescents and adults leaving early childhood immunizations to the primary care provider (PCP). Oftentimes, allergy patients simply do not take care to see both their $\mathrm{A} / \mathrm{I}$ as well as their primary care provider. It thus falls on the $\mathrm{A} / \mathrm{I}$ specialist to provide the missing immunizations. Simple patient screening using basic questionnaires regarding age-related vaccinations is appropriate and can quickly establish which vaccinations the patient is missing. A/I offices are well-equipped to provide the following eight vaccinations: influenza, pneumococcal polysaccharide and conjugate, Tdap, shingles, meningococcal ACWY and B, and human papilloma virus. Russell and Tankersley have recently published the rationale and economic feasibility of administering the shingles vaccine in an allergy private practice [31•].

Good traffic habits in the exam room can also save time. Basic preparedness can include having all necessary patient paperwork such as written patient instructions, asthma action plans, educational handouts, lab, radiology, and sleep study order forms, and immunotherapy consent forms present in the office binder or notebook to reduce delays as well as patient overall length of stay [32].

Other good organization practices also involve going paperless, such as increasing use of patient portals and HIPPAcompliant email systems to transmit immunization records, new patient packets and account statements. Utilizing reusable laminated encounter forms for new patient paperwork or screening questionnaires might also be implemented. On the other hand, knowing when to maintain paper records for immunotherapy can potentially save thousands of dollars per year for a solo allergist, who may not have the patient numbers to justify purchasing costly immunotherapy-specific EHRs.

Table 2 T.R.U.E. TEST compared to American Core Allergen Series

\begin{tabular}{ll}
\hline T.R.U.E. TEST TM & American Core Allergen Series ${ }^{\mathrm{TM}}$ \\
\hline$\$ 3.85$ per allergen & $\$ 0.32$ per allergen \\
36 allergens per test & 80 allergens in series \\
Cost: $\$ 3.85 \times 36=\$ 138.60$ & Cost: $\$ 0.32 \times 80=\$ 25.60$ \\
Profit margin: $\$ 55$ per patient & Profit margin: $\$ 406.40$ per patient \\
Nursing time: minimal & Nursing time: 30 min $(\sim \$ 10)$ \\
\hline
\end{tabular}

EHRs can potentially include templates that allergists can utilize to avoid missing important details of a patient's past medical history. This can standardize the evaluation of new patients and can transfer easily into the EHR. This would include the history of present illness regarding rhinitis, asthma, dermatitis, urticarial, or food or venom reactions and can allow the provider to have more face-to face time with the patient.

In addition to the office efficiencies mentioned above, proper coding of time consuming or complex patients based on time can maximize revenue and incentivizes spending the proper amount of time with each patient. Including a templated statement in the medical chart or EHR documenting the time spend with the patient can allow for efficient coding. An example of such a statement would be, "Greater than $50 \%$ of the $80 \mathrm{~min}$ appointment consisted of the coordination of care and education of the condition to include discussions of the following: diagnostic results, prognosis, risks and benefits of treatment options, impressions, instructions for management, importance of compliance with chosen treatment options, risk factor reductions, and patient and family education."

Expanding patient access to various products not commonly used in allergy clinics can be an important tool to increase patient satisfaction, provide quality medical care, and increase office revenue. Patch testing for allergic contact dermatitis has become a niche practice that can be easily incorporated into busy practices with the help of patch testing products such as

Table 3 List of additional workflow efficiencies to improve clinical practice $[1,8$, $24 \cdot, 31 \cdot, 32]$
1. Hire a scribe

2. Use a cost efficient EHR

3. Expand immunization product line

4. Hire family members

5. Expand patch testing product line

6. Go paperless

7. Do your own billing

8. Utilize AAAAI practice management solution

9. Attend AAAAI practice management workshop

10. Brand your practice 
the T.R.U.E test from SmartPractice or the American Core Allergen Series (ACAS). T.R.U.E test standardizes and serializes common allergens into easily applied tests but are costly to purchase. ACAS ( 80 allergens) is a more comprehensive panel of the 80 most common contact allergens with a tenfold less cost than the T.R.U.E test so also more cost-effective to integrate into an allergy practice. Currently, unlike skin prick testing or spirometry, few allergists offer the comprehensive ACAS patch testing panel. However, as patch testing has a similar reimbursement of allergy prick skin testing and can provide patients with needed information to dramatically improve quality of life due to allergic contact dermatitis, we suggest that all allergists consider implementing the more comprehensive and more cost-effective ACAS patch testing panel as part of a complete evaluation. Further details comparing both the cost and time needed to apply each patchtesting product can be reviewed in Table 2 .

Other workflow efficiencies to further improve clinical care include offering new patients the ability to choose a cluster protocol for initiation of subcutaneous immunotherapy (SCIT) in order to reach maintenance dosing faster in fewer visits. Studies to date have demonstrated that this method is relatively as safe as traditional build-up methods [33, 34]. Using CPT 95180, two cluster immunotherapy injections given $30 \mathrm{~min}$ apart reimburses around sevenfold per injection that of a conventional immunotherapy CPT 95115/95117.

In the outpatient allergy setting as many patients do not have a primary care provider, administer a Patient Health Questionnaire-9 (PHQ-9) to screen annually for depression as recommended nationally. Unfortunately, there are on average 123 suicides per day in the USA, and as a part of the healthcare team it is important to screen our patients for comorbid conditions such as depression and anxiety [35]. This is coded with CPT G0444 with an associated ICD-10 of Z13.31 with an annotation of $15 \mathrm{~min}$ for time.

Other ways to improve workflow efficiencies in your clinical operations include implementing an adherence tracking system for all SCIT patients. By implementing a tracking system, it is easier to know exactly how many patients receive SCIT and to keep track of needed follow-up visits as these visits can easily fall off the radar (Table 3) [36].

\section{Conclusion}

In the ever-changing healthcare system along with new advancements in the field of allergy, the workflow for the allergist continues to evolve requiring more time spent doing nonclinical duties such as documentation and reviewing reimbursement challenges in the midst of busy clinics. With increasing demands placed on the allergist daily, it is imperative the allergist find ways to improve workflow efficiency. By utilizing electronic health records, scribes, and exploring other areas to improve workflow efficiency, allergists will be able to meet the demands of the healthcare system and still provide patients with evidence-based, compassionate, and costeffective care.

Availability of Data and Material N/A

\section{Compliance with Ethical Standards}

Conflict of Interest Dr. Tankersley is a speaker and on the advisory board for ALK. The other authors declare no conflicts of interest relevant to this manuscript.

Human and Animal Rights and Informed Consent This article does not contain any studies with human or animal subjects performed by any of the authors.

Code Availability N/A

\section{References}

Papers of particular interest, published recently, have been highlighted as:

- Of importance

1. Rathkopf M, Hare N. Electronic medical records, electronic prescribing, mobile technology and practice management software. AAAAI Pract Manag Resource Guid. 2014:1-22 https://www. aaaai.org/Aaaai/media/MediaLibrary/PDF/Documents/Practice/ Management/PM/Resource/Guide/Chapter-10-EMRs-and-mobiletechnology.pdf. Accessed 21 June 2020.

2. Atherton J. History of medicine: development of the electronic health record. AMA J Ethics. 2011;13(3):186-9.

3. The American Recovery and Reinvestment Act of 2009. 2009. https://www.ntia.doc.gov/page/2011/american-recovery-andreinvestment-act-2009. Date accessed 07 March 2020.

4. Frenkel L. Electronic health records-applications for the allergist/ immunologist: all that glitters is not gold. Allergy Asthma Proc. 2016;37(4):273-8.

5. Menachemi N, Collum T. Benefits and drawbacks of electronic health record systems. Risk Manag Healthc Policy. 2011;4:49-55.

6. Bates DW, Leape LL, Cullen DJ, Laird N, Petersen LA, Teich JM, et al. Effect of computerized physician order entry and a team intervention on prevention of serious medication errors. J Am Med Assoc. 1998;280(15):1311-6.

7. Bates DW, Teich JM, Lee J, Seger D, Kuperman GJ, Ma'Luf N, et al. The impact of computerized physician order entry on medication error prevention. J Am Med Inform Assoc. 1999;6(4):31321.

8. King J, Patel V, Jamoom EW, Furukawa MF. Clinical benefits of electronic health record use: national findings. Health Serv Res. 2014;49(2):392-404 An Important study illustrating cost saving benefits of electronic health record use.

9. Ledwich LJ, Harrington TM, Ayoub WT, Sartorius JA, Newman ED. Improved influenza and pneumococcal vaccination in rheumatology patients taking immunosuppressants using an electronic health record best practice alert. Arthritis Rheum. 2009;61(11): 1505-10.

10. Kucher N, Koo S, Quiroz R, Cooper JM, Paterno MD, Soukonnikov B, et al. Electronic alerts to prevent venous 
thromboembolism among hospitalized patients. N Engl J Med. 2005;352(10):969-77.

11. McDonald CJ, Hui SL, Tierney WM. Effects of computer reminders for influenza vaccination on morbidity during influenza epidemics. MD Comput. 1992;9(5):304-12.

12. Makam AN, Lanham HJ, Batchelor K, et al. The good, the bad and the early adopters: providers' attitudes about a common, commercial EHR. J Eval Clin Pract. 2014;20(1):36-42.

13. Alkureishi M, Wei Wei L, Lyons M. Impact of electronic medical record use on the patient-doctor relationship and communication: a systematic review. J Gen Intern Med. 2016;31(5):548-60.

14. Zeiger RS, Schatz M, Li Q, Solari PG, Zazzali JL, Chen W. Realtime asthma outreach reduces excessive short-acting beta-agonist use: a randomized study. J Allergy Clin Immunol Pract. 2014;2: 445-456.e5.

15. Blumenthal K, Park M, Macy E. Redesigning the allergy module of the electronic health record. Ann Allergy Asthma Immunol. 2016;17:126-31.

16. Cowie M, Blomster J, Curtis L, et al. Electronic health record to facilitate clinical research. Clin Res Cardiol. 2017;106:1-9.

17. Coorevits P, Sundgren M, Klein GO, Bahr A, Claerhout B, Daniel $\mathrm{C}$, et al. Electronic health records: new opportunities for clinical research. J Intern Med. 2013;274:547-60.

18. Cheng D, Scodellaro T, Uahwatanasakal W, South M. An electronic medical record in pediatric medical education: survey of medial students' expectations and experiences. Appl Clin Inform. 2018;9: 809-16.

19. Fleming NS, Becker ER, Culler SD, et al. The impact of electronic health records on workflow and financial measures in primary care practices. Health Serv Res. 2014;49(1 Pt 2):405-20.

20. Practice Fusion - \#1 Cloud-Based Electronic Health Record. 2020. https://practicefusion.com/electronic-health-record-ehr/. Accessed 14 April 2020.

21. Gao R, et al. Effect of medical scribes on outpatient oncology visits at a multidisciplinary Cancer center. JCO Oncol Pract. 2020;16: e139-47.

22. Cancian M, Pareek G, schiff S, Thavaseelan S. Scribes in ambulatory urologic practice: financial analysis and practice management considerations. J Urol. 2017;197. https://doi.org/10.1016/j/juro. 2017.02.2141.

23. Salzberg L. Physician well-being: improving office efficiency. F P Essent. 2018;471:16-9.

24. Walker K, et al. Impact of scribes on emergency medicine doctors' productivity and patient throughput: multicentre randomised trial.
BMJ. 2019;364:1121 A thorough study illustrating cost saving, burnout avoidance, and benefits of scribes.

25. McCormick BJ, Deal A, Borawski KM, Raynor MC, Viprakasit D, Wallen EM, et al. Implementation of medical scribes in an academic urology practice: an analysis of productivity, revenue, and satisfaction. World J Urol. 2018;36:1691-7.

26. Pozdnyakova A, Laiteerapong N, Volerman A, Feld LD, Wan W, Burnet DL, et al. Impact of medical scribes on physician and patient satisfaction in primary care. J Gen Intern Med. 2018;33:1109-15.

27. Shultz CG. The use of medical scribes in health care settings: a systematic review and future directions. J Am Board Fam Med. 28(3):371-81. https://doi.org/10.3122/jabfm.2015.03.140224.

28. Bastani A, Shaqiri B, Palomba K, Bananno D, Anderson W. An ED scribe program is able to improve throughput time and patient satisfaction. Am J Emerg Med. 2014;32:399-402.

29. Taylor L, Waller M, Portnoy J. Telemedicine for allergy services to rural communities. J Allergy Clin Immunol Pract. 2019;7(8):2554 9.

30. Doxy.me: The simple, free, and secure telemedicine solution. 2020. https://doxy.me/. Accessed 5 May 2020.

31. Russell HG, Tankersley MS. Recombinant zoster vaccine administration in an allergy and immunology practice. Ann Allergy Asthma Immunol. 2020;124(5):517-8 An important study illustrating the ease with which practicing allergists can implement recommended adult vaccines into their daily routine.

32. Russell HG, Tankersley MS. Increasing clinic efficiency: lean allergy. J Allergy Clin Immunol Pract. 2019;7(8):2946-7.

33. dos Reis Pimentel RAG, Oliveira G, Loureiro e Lemos CSFC. Accelerated subcutaneous immunotherapy in pediatric population - systematic review. Pulmonology. 2018;24(3):182-93.

34. Winslow AW, et al. Comparison of systemic reactions in rush, cluster, and standard-build aeroallergen immunotherapy. Ann Allergy Asthma Immunol. 2016;117(5):542-5.

35. Suicide Statistics. American Foundation for Suicide Prevention. 2020. https://afsp.org/about-suicide/suicide-statistics/. Accessed 10 March 2020

36. Russell HG, Tankersley MS. SCIT adherence: making IT happen! J Allergy Clin Immunol Pract. 2019;7(7):2505-6.

Publisher's Note Springer Nature remains neutral with regard to jurisdictional claims in published maps and institutional affiliations. 